\title{
Estriol Vaginal Gel
}

National Cancer Institute

\section{Source}

National Cancer Institute. Estriol Vaginal Gel. NCI Thesaurus. Code C121779.

A vaginal gel formulation containing a very low concentration of the estrogen estriol, that can be used for hormone replacement. Upon topical application to the vagina, estriol maintains adequate levels of estrogen in the vagina, and decreases vaginal atrophy and its associated symptoms, such as vaginal dryness and itching. 